\title{
INTEGRAL and XMM-Newton observations of the puzzling binary system LSI +61303
}

\author{
Masha Chernyakova* \\ ISDC Versoix, Switzerland \\ Geneva Observatory, University of Geneva, Sauverny, Switzerland \\ E-mail: masha.chernyakova@obs.unige.ch
}

\section{Andrii Neronov}

ISDC Versoix, Switzerland

Geneva Observatory, University of Geneva, Sauverny, Switzerland

E-mail: andrii.neronov@obs.unige.ch

\section{Roland Walter}

ISDC Versoix, Switzerland

Geneva Observatory, University of Geneva, Sauverny, Switzerland

E-mail: roland.walter@obs.unige.ch

LSI $+61^{\circ} 303$ is one of the few X-ray binaries with Be star companion from which both radio and high-energy gamma-ray emission have been observed. We present XMM-Newton and INTE$G R A L$ observations which reveal variability of the X-ray spectral index of the system. The X-ray spectrum is hard (photon index $\Gamma \simeq 1.5$ ) during the orbital phases of both high and low X-ray flux. However, the spectrum softens at the moment of transition from high to low X-ray state. The spectrum of the system in the hard X-ray band does not reveal the presence of a cut-off (or, at least a spectral break) at 10-60 keV energies, expected if the compact object is an accreting neutron star. The observed spectrum and spectral variability can be explained if the compact object in the system is a rotation powered pulsar. In this case the recently found X-ray spectral variability of the system on the several kiloseconds time scale can be explained by the clumpy structure of the Be star disk.

VI Microquasar Workshop: Microquasars and Beyond September 18-22, 2006

Como, Italy

\footnotetext{
${ }^{*}$ Speaker.
} 


\section{Introduction}

The Be star binary LSI $+61^{\circ} 303$ is known to be a source of variable optical, radio, X-ray, gamma-ray and very high-energy gamma-ray emission. The periodicity of $T=26.4960$ days of radio emission was associated with the binary orbital period (Gregory et al., 2002). Optical data allow to constrain the orbital parameters of the system revealing the eccentricity of the orbit, $e \simeq$ 0.7 (Casares et al., 2005). However, the measurements are not sufficient to determine the nature of the compact object (neutron star or black hole), because the inclination of the orbit is poorly constrained. Radio observations reveal the presence of $100 \mathrm{AU}$-scale jet in the system which places LSI $+61^{\circ} 303$ among the Galactic micro-quasars (Massi et al., 1993, 2004). The system is also a Galactic "micro-blazar" due to its association with $100 \mathrm{MeV}$ gamma-ray source 2CG 135+01 (Tavani et al., 1998) visible up to $\mathrm{TeV}$ energies (Albert et al., 2006). The spectral and timing properties of the system in different energy ranges is summarized in Figures 1 and 2.

Below we present a study of LSI $+61^{\circ} 303$ in the $0.5-100 \mathrm{keV}$ energy band with XMM-Newton and INTEGRAL and discuss the observed spectral variability on the different time scales.

\section{Observations}

XMM-Newton has observed LSI $+61^{\circ} 303$ with the EPIC instruments five times during 2002, and once in 2005. Four 2002 observations have been done during the same orbital cycle, and the fifth one has been done seven months later. A simple power law with photoelectric absorption $\left(N_{H}=0.49 \times 10^{22} \mathrm{~cm}^{-2}\right)$ describes the spectrum of LSI $+61^{\circ} 303$ well, with no evidence for any line features. The details of data analysis are given in Chernyakova et al. (2006); Sidoli et al. (2006). The log of the XMM-Newton data along with the spectral characteristics is presented in Table 1.

For INTEGRAL analysis we have used all available public data spread over the period from the January 2003 (rev 25) until March 2005 (rev 288). Overall we have analyzed 600 science windows which resulted in an effective vignetting corrected exposure of $273 \mathrm{ksec}$. The averaged spectrum of the source in hard X-rays $(20-100 \mathrm{keV})$ is described by a simple power law $(\Gamma=1.6 \pm 0.2$, $F_{20-60}=2.5 \pm 0.3 \times 10^{-11} \mathrm{ergs} \mathrm{s}^{-1} \mathrm{~cm}^{-2}$ ), see Chernyakova et al. (2006).

Table 1: Journal of XMM-Newton observations of LSI $+61^{\circ} 303$

\begin{tabular}{lcccccc}
\hline $\begin{array}{c}\text { Data } \\
\text { Set }\end{array}$ & Date & $\begin{array}{c}\text { Orbital } \\
\text { Phase }\end{array}$ & $\begin{array}{c}\text { Exposure } \\
(\mathrm{ks})\end{array}$ & $\begin{array}{c}F(2-10 \mathrm{keV}) \\
10^{-11} \mathrm{erg} \mathrm{s}^{-1}\end{array}$ & $\Gamma$ & $\chi^{2}$ (dof) \\
\hline X1 2002-02-05 & 0.55 & 6.4 & $1.30 \pm 0.04$ & $1.60 \pm 0.03$ & $474(456)$ \\
X2 2002-02-10 & 0.76 & 6.4 & $1.24 \pm 0.04$ & $1.54 \pm 0.03$ & $440(446)$ \\
X3 2002-02-17 & 0.01 & 6.4 & $0.61 \pm 0.03$ & $1.78 \pm 0.04$ & $184(188)$ \\
X4 2002-02-21 & 0.18 & 7.5 & $0.44 \pm 0.03$ & $1.52 \pm 0.06$ & $137(150)$ \\
X5 2002-09-16 & 0.97 & 6.4 & $1.25 \pm 0.05$ & $1.55 \pm 0.02$ & $488(496)$ \\
X6a 2005-01-27 & 0.61 & 16.0 & $1.29 \pm 0.01$ & $1.62 \pm 0.01$ & $681(596)$ \\
X6b 2005-01-28 & 0.61 & 29.0 & $0.40 \pm 0.01$ & $1.83 \pm 0.01$ & $618(533)$ \\
\hline
\end{tabular}




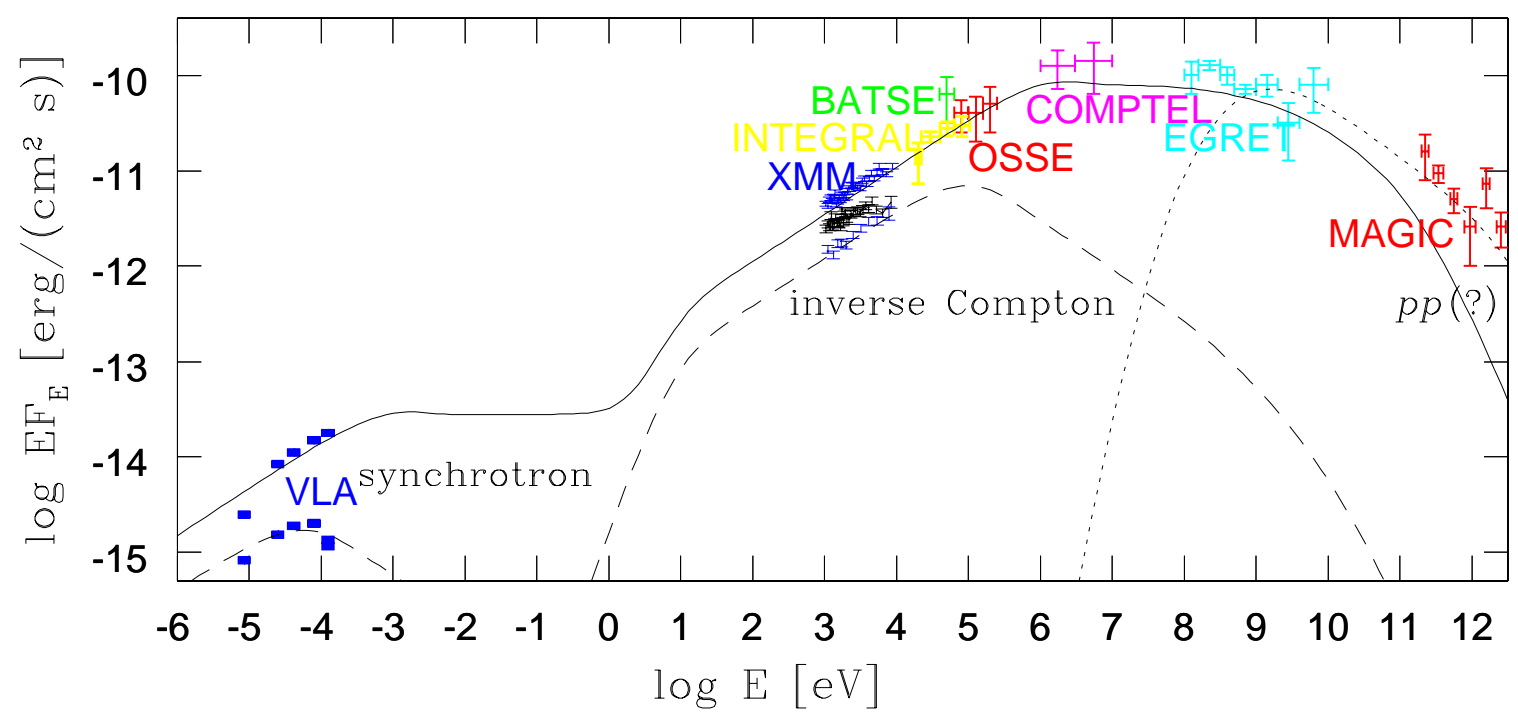

Figure 1: Broad band spectrum of LSI $+61^{\circ} 303$. Radio data points are taken from Strickman et al. (1998). CGRO data points are from Tavani et al. (1998). MAGIC data points are from Albert et al. (2006). The solid (dashed) line shows the model fit within the synchrotron-inverse Compton model for the high (low) flux state of the source. The dotted line shows possible contribution from the proton proton interactions. The values of parameters of the model fits are cited in the text.

\section{Broad band spectrum of the source.}

Historically there are two broad classes of physical models of the source activity. The first one assumes that the activity is powered by accretion onto the compact object (either neutron star or a black hole) (Taylor \& Gregory, 1984). Most of the Be star X-ray binaries contain an accreting neutron star as the compact object and most of them are transient sources. The spectra of these sources are characterized by the presence of an exponential cut-off in the hard X-ray band at energies 10-60 keV (Filippova et al., 2005). Our analysis of the INTEGRAL data shows that in the case of LSI $+61^{\circ} 303$ no high-energy cut-off is found at the energies below $100 \mathrm{keV}$, the fact that does not fit well into the "conventional" accretion scenario.

In the second class of models, first proposed by Maraschi \& Treves (1981), the activity of the source is explained by interactions of a young rotation powered pulsar with the wind from the companion Be star. Contrary to accreting compact object models, a featureless powerlaw keV $\mathrm{MeV}$ spectrum is expected in the "rotation powered pulsar" scenario. In this scenario, radio, X-ray and gamma-ray emission come from the region where the pulsar wind interacts with the wind of $\mathrm{Be}$ star. An example of synchrotron - inverse Compton model fit of the broad band (radio to gammaray) spectrum of the source is shown in Fig. 1. The model parameters used for the fits of the broad band spectrum of the system in high (low) flux state in Fig. 1 are $B=0.35 \mathrm{G}(B=0.25 \mathrm{G})$, the electron spectrum described by a broken powerlaw with the spectral index $\Gamma_{e}=2$ below the break energy $E_{b r}=2 \times 10^{8} \mathrm{eV}\left(E_{b r}=10^{8} \mathrm{eV}\right)$ and $\Gamma_{e}=3.5\left(\Gamma_{e}=4\right)$ above the break energy. Such a break in the electron spectrum is natural if the relativistic electrons responsible for the X-ray emission are initially injected at high energies and subsequently cool down forming the characteristic $E^{-2}$ cooling spectrum. 

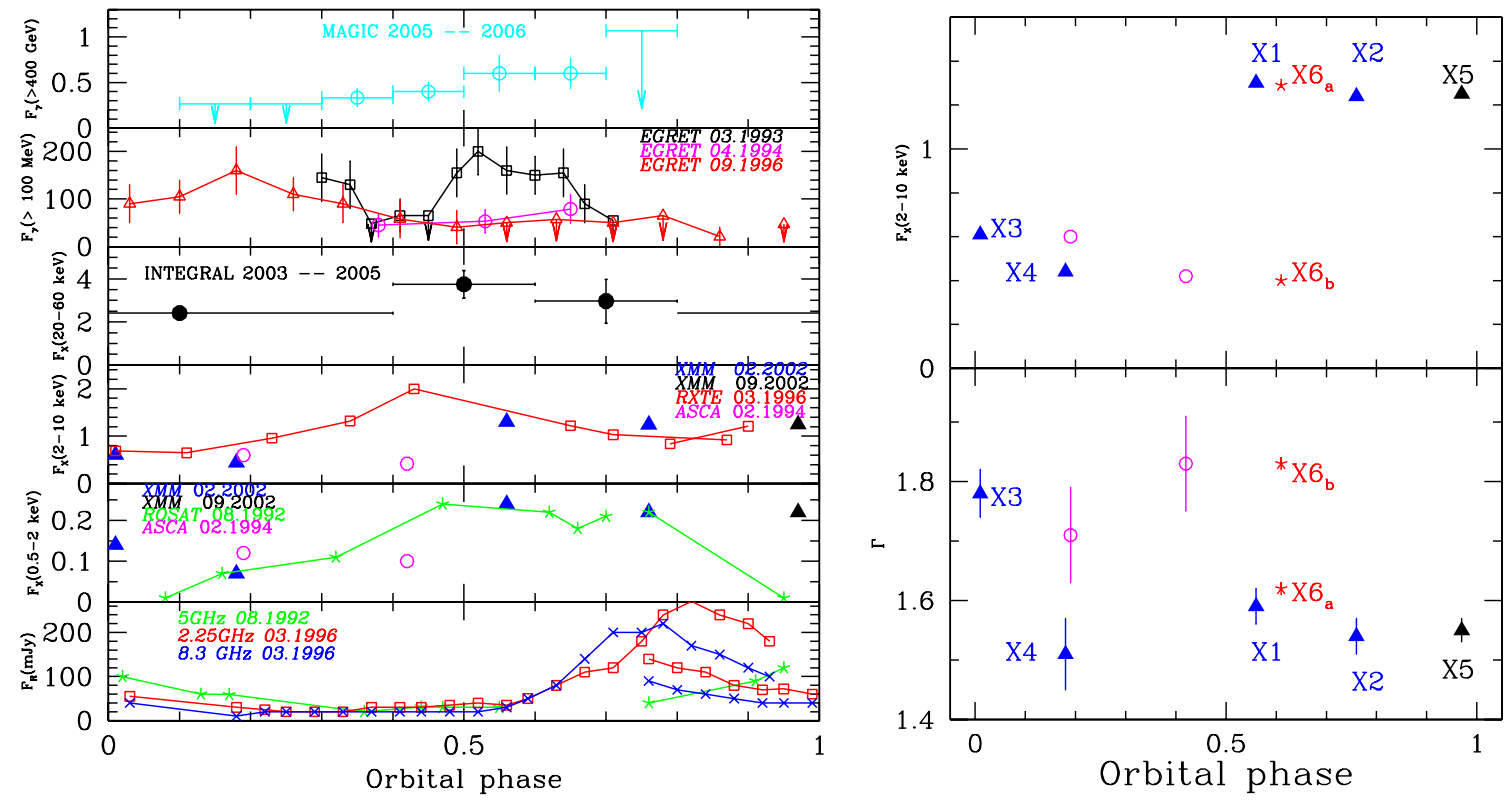

Figure 2: Left panel: Comparison between the $\mathrm{TeV}$ (top), $\mathrm{GeV}$ gamma-ray orbital lightcurves of LSI $+61^{\circ} 303$ with the hard X-ray (20-60 keV), X-ray (2-10 keV), soft X-ray $(0.5-2 \mathrm{keV})$ and radio (bottom) orbital lightcurves. The $0.5-2 \mathrm{keV}$ and $2-10 \mathrm{keV} \mathrm{X}$-ray flux is given in $10^{-11} \mathrm{ergs} \mathrm{cm}^{-2} \mathrm{~s}^{-1}$. GeV $\gamma$-ray flux $(>100 \mathrm{MeV})$ is given in the units of $10^{-8} \mathrm{ph} \mathrm{cm}^{-2} \mathrm{~s}^{-1}$. TeV flux $(>400 \mathrm{GeV})$ is in the units of $10^{-11} \mathrm{ph} \mathrm{cm}^{-2} \mathrm{~s}^{-1}$ ). To guide the eye we have connected with lines data from the same orbital cycle. The time is assumed to increase from left to right, thus if the observation has started at the end of the orbital cycle it is shown with two lines of the same color, the line starting at larger orbital phase connects data points taken earlier. References for all data points are given in Chernyakova et al. (2006). Right panel: Spectral variability of the system in 2-10 keV energy range, as observed with XMM-Newton and ASCA (open circles).

\section{Spectral variability of the source.}

The graphical representation of the evolution of the spectral parameters along the orbit is given on the right panel of Figure 2. In the XMM-Newton energy band the powerlaw photon index is $\Gamma \simeq 1.5$ in all 2002 observations except for the observation $\mathrm{X} 3$, where we find significantly softer spectrum with $\Gamma=1.78 \pm 0.04$. This indicates that the $\mathrm{X}$-ray spectrum softens during the transition from higher to lower flux state. The softening of the spectrum can be explained assuming that the injection of electrons at high energies drops and higher energy electrons (emitting in the hard X-ray band) cool faster than the low energy ones (emitting in the soft X-ray band).

Injection from high energies can be explained if the electrons responsible for the X-ray emission originate from the cold pulsar wind with bulk Lorentz factor $\geq($ several $) \times 10^{2}$. In this case all electrons have initial energies larger than $100 \mathrm{MeV}$ and the electron spectrum below $100 \mathrm{MeV}$ formed in the process of inverse Compton cooling has the spectral index $\Gamma_{e} \simeq 2$. However, an immediate difficulty with such simple injection model is that the injection rate is not expected to vary with time, contrary to what is observed.

The variable injection rate could be provided if relativistic protons are either present in the pulsar wind or accelerated in the shock at the contact surface of pulsar and stellar wind. Such 
relativistic protons could interact with the low energy protons from the disk and produce injection of electrons at energies above $\sim 100 \mathrm{MeV}$ via production and subsequent decays of charged pions. In this case variable injection rate of high-energy electrons is explained by the variations in the density of the stellar wind protons along the pulsar orbit.

The 2005 XMM-Newton observations reveal X-ray spectral variability of the system on the several kiloseconds time scale (Sidoli et al., 2006). This time scale coincides with the Compton cooling time $t_{i c}=5\left(10^{38} / L_{*}\right)\left(R / 10^{12}\right)^{2}\left(E_{I C} / 1 \mathrm{KeV}\right)^{0.5} \mathrm{ks}$, and in the frame of the proton-proton interaction model can be explained by the presence of the clumps in the wind of the Be star. Indeed, if the pulsar wind meets a dense clump on its way it will lead to the higher injection rate of the relativistic particles, which will decrease after the passage through the clump, leading to the total intensity decrease and softening of the spectrum in agreement with the observations. The size of the clump can be estimated as $R_{\text {clump }} \sim v_{p} \Delta t \sim 10^{11} \mathrm{~cm}$ (here $v_{p}$ is the pulsar relative velocity, and $\Delta t$ is the variability time).

A straightforward consequence of high-energy proton interactions in the Be star disk is the appearance of additional component in the high-energy $\gamma$-ray spectrum, resulting from the two photon decays of neutral pions. The spectrum of the pion decay gamma-rays in the GeV-TeV energy band has the same spectral index as the spectrum of the high-energy protons. In Fig. 1 we show possible contribution to the $\gamma$-ray spectrum of the source which can be produced in result of proton-proton interactions.

\section{References}

Albert J., et al., 2006, Science, 312, 1771

Casares J., Ribas I., Paredes J.M. et al., 2005, MNRAS, 360, 1105

Chernyakova M., Neronov A., Walter R., 2006, MNRAS, in press [astro-ph/0606070].

Filippova, E. V., Tsygankov, S. S., Lutovinov, A. A., Sunyaev, R. A., 2005, Astr. Lett., 31, 729.

Gregory P.C. 2002, ApJ, 575, 427

Massi M., Paredes J.M., Estalella R., Felli M., 1993, A\&A, 269, 249

Massi M., Ribo M., Paredes J.M. et al., 2004, A\&A, 414, L1.

Maraschi L., Treves A., 1981, MNRAS, 194, 1P

Sidoli L., Pellizzoni A., Vercellone S. et al., A\&A, in press [astro-ph/0606307]

Strickman M. S., Tavani M., Coe M. J. et al., 1998, Ap.J. 497, 419.

Tavani M., Kniffen D., Mattox J.R. et al., 1998, ApJ, 497, L89

Taylor A.R., Gregory P.C., 1984, ApJ, 283, 273 